\title{
Stabilisation of Self-Assembled DNA Crystals by Triplex-Directed Photo-Cross-Linking
}

\author{
Hatem O. Abdallah, ${ }^{a}$ Yoel P. Ohayon, ${ }^{a}$ Arun Richard Chandrasekaran, ${ }^{a, b}$ Ruojie Sha, ${ }^{a}$ Keith R. Fox,${ }^{c}$ \\ Tom Brown, ${ }^{d}$ David A. Rusling, ${ }^{c}$ Chengde Mao ${ }^{d}$ and Nadrian C. Seeman ${ }^{a}$ \\ Received (in $X X X, X X X)$ Xth $X X X X X X X X X 20 X X$, Accepted Xth $X X X X X X X X X 20 X X$ \\ DOI: $10.1039 / b 000000 x$
}

The tensegrity triangle is a robust DNA motif that can selfassemble to generate macroscopic three-dimensional crystals. However, the stability of these crystals is dependent on the 10 high ionic conditions used for crystal growth. Here we demonstrate that a triplex-forming oligonucleotide can be used to direct the specific intercalation, and subsequent photo-cross-linking, of 4,5',8-trimethylpsoralen to single or multiple loci within or between the tiles of the crystal. Crossis linking between the tiles of the crystal improves their thermal stability. Such an approach is likely to facilitate the removal of crystals from their mother liquor and may prove useful for applications that require greater crystal stability.

The tensegrity triangle is composed of three double-helices 20 directed along linearly independent vectors. ${ }^{1 \mathrm{a}}$ By tailing the helices with complementary single-stranded overhangs, each triangle can associate with six others, yielding three-dimensional (3D) DNA crystals. ${ }^{1 b}$ Such structures allow the programmable positioning of components in 3D-space and offer applications that ${ }_{25}$ include the organisation of nanoelectronics, ${ }^{2 a}$ the direction of biochemical and chemical cascades, ${ }^{2 \mathrm{~b}}$ and the structure determination of periodically positioned molecules for X-ray diffraction analysis. ${ }^{2 \mathrm{c}}$ However, in some cases the applications of these crystals are limited by the crystallisation process; their 30 removal into a physiological, or other relevant buffer, is hampered, since their formation relies on reversible WatsonCrick (W-C) base pairing that is stabilised by the increase in ionic strength during crystallisation. One method to increase duplex stability is to cross-link the underlying strands using the photo35 cross-linking agent 4,5',8-trimethylpsoralen (psoralen). Psoralen intercalation at $\mathrm{TpA}$ steps results in a $2+2$ cycloaddition reaction with the adjacent thymidines upon UV exposure, thereby crosslinking the two duplex strands. ${ }^{3}$ Indeed psoralen has already been used to increase the stability of nanostructures assembled by ${ }_{40}$ DNA origami. ${ }^{4}$ However, this approach will not be tolerated well by the tensegrity triangle since multiple intercalation events will unwind the DNA and disrupt the precise crossover positioning required for tile and/or crystal assembly. Moreover, since intercalation occurs preferentially at any pyrimidine-purine 45 $(\mathrm{YpR})$ step the cross-linking reaction is not easily controlled. Here we demonstrate that the triplex approach to DNA recognition $^{5 \mathrm{a}-\mathrm{c}}$ can be used to overcome this limitation by directing the specific intercalation, and subsequent photo-crosslinking, of psoralen to unique loci within or between the tiles of ${ }_{50}$ the tensegrity triangle crystal (Fig. 1).

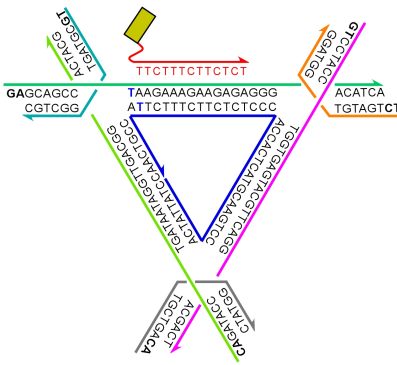

3TA-intra

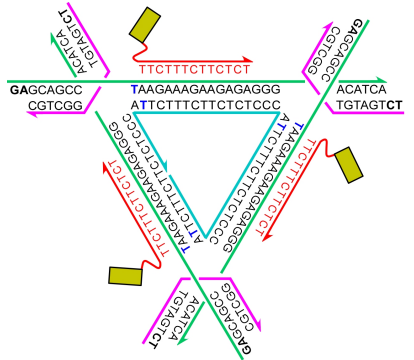

3TS-intra
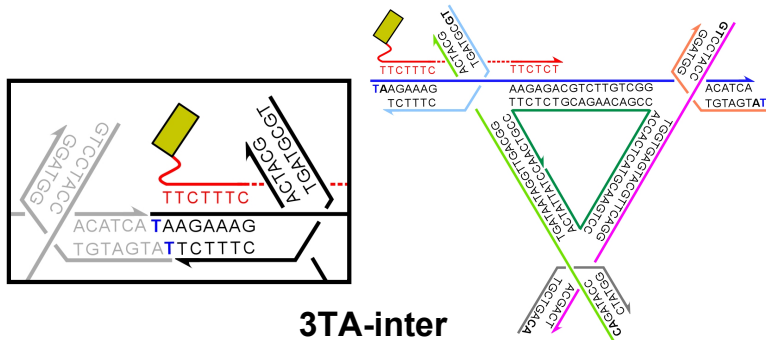

Fig. 1. Sequence of the tiles and TFOs used in this study. Duplex sequences are shown in black, TFO in red, psoralen in yellow and the thymidines targeted for cross-linking in dark blue. For the 3TA-inter tile $s$ the TFO will cross-link TpA steps only generated upon the association of two or more adjacent tiles within a crystal (inset). For reference full oligonucleotide sequences are included in Fig. S1 (ESI $\dagger$ ).

We have previously demonstrated that triplex-forming 60 oligonucleotides (TFOs) can be used to target and introduce components at specific locations within a tensegrity triangle crystal under slightly acidic conditions. ${ }^{6}$ TFOs bind within the duplex major groove by generating $\mathrm{C}^{+}-\mathrm{GC}$ and T-AT base triplets when an appropriate oligopurine-oligopyrimidine sequence is ${ }_{5}$ embedded between crossovers of the triangle. ${ }^{5 \mathrm{a}, \mathrm{b}, \mathrm{c}}$ The attachment of a dye to the oligonucleotide leads to its targeted introduction at these sites. The same strategy is used here but this time the TFO is used to direct the intercalation of a tethered psoralen molecule to a TpA step introduced adjacent to the TFO target site (Fig. 1). ${ }^{7}$ ${ }_{70}$ Three systems were designed to examine the cross-linking of single (3-turn asymmetric; 3TA-intra) or multiple TpA steps (3turn symmetric; 3TS-intra) within each tile, as well as the cross- 
linking of a TpA step generated only upon the association of adjacent tiles of the crystal (3TA-inter) via sticky-ended cohesion. The 3TA-intra and 3TA-inter tiles are asymmetric systems generated from seven unique strands and include a single 5 TFO target sequence on one helix; the 3TS-intra tile is a threefold rotationally symmetric system, assembled from three strands in a 3:3:1 ratio and includes a TFO target sequence on each of the three helices. In each case, the helical edge of the tile is 31 nucleotides (nts); approximately three helical turns. We chose the

10 three-turn system because it can be assembled with 31 or 32 base pairs per helical edge suggesting one intercalation event can be accommodated per helix without undue stress on the twist of the system. ${ }^{\mathrm{lb}}$ In addition, crystals assembled from three-turn triangles generate rhombohedral cavities that are likely to be large enough

15 to host macromolecules such as proteins (c.a. $\left.366 \mathrm{~nm}^{3}\right) .^{1 \mathrm{~b}}$ Stabilisation of such a system may therefore prove useful for different applications.

We first determined by non-denaturing polyacrylamide gel electrophoresis (PAGE) that both an unmodified TFO and a ${ }_{20}$ psoralen-modified TFO (pso-TFO) were capable of binding to each of the tiles in solution (Fig. S2). In each case, a single complex with reduced mobility was observed in the presence of the oligonucleotide, suggesting the TFOs were capable of binding to their intended target sites. We next examined whether the pso${ }_{25}$ TFO was capable of directing the specific photo-cross-linking of psoralen to the TpA step located adjacent to the 5' end of the TFO target sequence. The complexes were exposed to UV irradiation at $365 \mathrm{~nm}$ and the products of the reaction separated by denaturing PAGE alongside appropriate controls. Experiments

30 were first undertaken on the 3TA-intra tile since the products of the cross-linking reactions are most easily characterised (Fig. 2). In the absence of the TFO and UV irradiation only bands corresponding to the denatured triangle strands can be seen (Lanes 2 and 3). The same was seen for the tile in the presence of 35 the unmodified TFO both before and after UV irradiation, since the oligonucleotide lacks the tethered psoralen (Lanes 4 and 5). By contrast, in the presence of the pso-TFO, three retarded species were generated, and as expected, only after UV exposure (Lanes 6 and 7).

40 Although it is not possible to characterise the size or structural features of the retarded complexes by comparison with a linear DNA marker (Lane 1) a Ferguson plot was used to demonstrate that the topology of the cross-linked products is different from that of the linear controls, with roughly twice the frictional drag

45 (Fig. S3). The three species are most likely attributable to the mono- and bis-adducts generated by cross-linking the thymidines located on either, or both of the helical or central strands of the double-helical edge (Fig. 2). ${ }^{7}$ Importantly, no other cross-linked products are observed, despite the presence of multiple TpA steps

${ }_{50}$ located in other regions of the triangle; TFO binding is thus a prerequisite for psoralen intercalation and cross-linking when the psoralen groups are attached to the TFO. By quantifying the decrease in band intensity of the denatured central strand of the complex, the efficiency of psoralen cross-linking simultaneously

55 to each strand of the duplex is estimated to be $60 \%$ under these conditions. However, this cross-linking efficiency was improved to around $85 \%$ by exposing the complexes to UV irradiation for longer time periods, or by increasing the concentration of the
TFO (Fig. S4). Experiments were also carried out on the 3TS60 intra tile that contains three binding sites and three TpA steps for cross-linking. The results obtained were similar to above but this time higher order complexes were also generated, owing to the presence of multiple cross-linking sites (Fig. S5). Cross-linking was not examined with the 3TA-inter tile since the required TpA ${ }_{65}$ step, and its complement, are not generated until after crystal assembly.

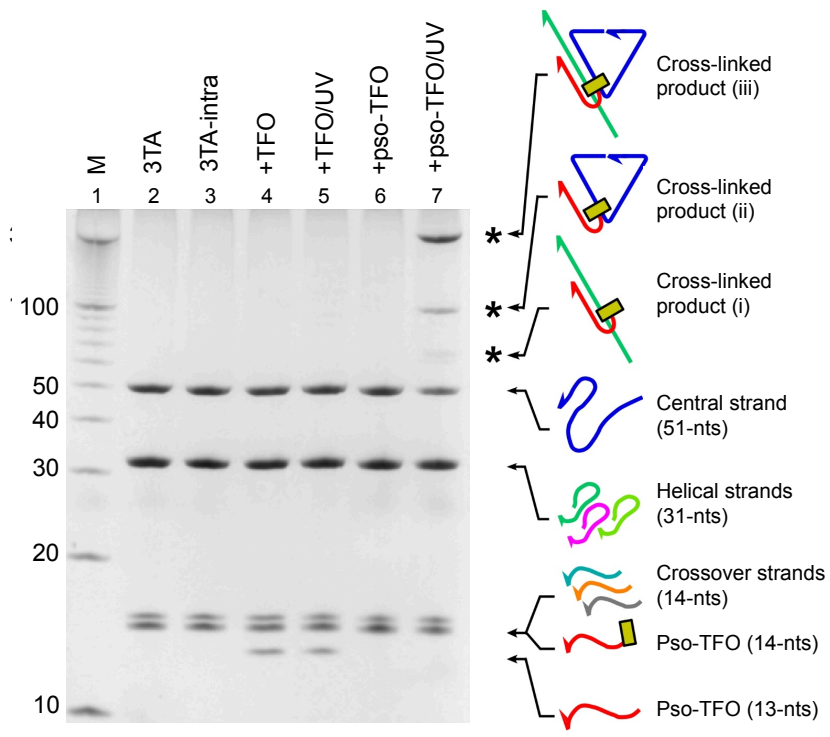

Fig. 2. Triplex-directed cross-linking of the tensegrity triangle in solution Tiles were annealed to $20^{\circ} \mathrm{C}$ before addition of the TFO. The complexes 70 were then exposed to UV light at $4{ }^{\circ} \mathrm{C}$ for one hour and the products separated by denaturing PAGE. The cartoon shows each of the denatured strands of the tile and products of the cross-linking reaction highlighted with an asterisk. Strand colourings are the same as those shown in Fig.

Since cross-linking between strands will improve the stability 75 of duplex regions within the tile this was examined by subjecting the tiles to thermal melting experiments before and after crosslinking (Fig. S6). Melting curves for all of the complexes exhibit two transitions: a transition at low temperature attributable to the dissociation of the shorter crossover strands located at the corners 80 of the triangle, followed by a transition at higher temperature for the dissociation of the central and helical strands that contain the TFO binding site. In the absence of TFO we find that the 3TSintra tile is more stable than the 3TA-intra tile with melting temperatures of $62{ }^{\circ} \mathrm{C}$ and $58{ }^{\circ} \mathrm{C}$ for the higher transitions, ${ }_{85}$ respectively. In the presence of the TFO and cross-linking each of these transitions is shifted to a higher temperature, demonstrating the increase in stability of the cross-linked complexes. As expected, the shift was greatest for the 3TS-intra tile that contains three cross-linked regions. The change in absorbance of the ${ }_{0}$ higher transition for this tile was also much less, suggesting that regions of the triangle do not fully dissociate, as expected. Melting temperatures measured for the 3TA-intra and 3TS-intra tiles were 62 and $73{ }^{\circ} \mathrm{C}$, an increase in 4 and $11{ }^{\circ} \mathrm{C}$, respectively. Cross-linking clearly increases the stability of the underlying 95 duplex regions within the tiles in solution.

To examine whether the cross-linking reaction could be achieved after crystal self-assembly, we crystallised each of the three tiles in turn with and without the pso-TFO, using the hanging drop vapour diffusion method. Figure 3 shows optical 
images of crystals obtained for the three tiles in the presence and absence of the psoralen TFO. The morphologies of the crystals are similar before and after TFO addition suggesting binding does not substantially disrupt the structure of the tile. ${ }^{6}$ Crystals were then subjected to UV irradiation for one hour to initiate crosslinking and no visible crystal degradation was evident (Fig. 3). Crystals were removed from the drop and washed to remove any tiles or TFOs left in solution. After heating the crystals the products of the cross-linking reactions were separated by 10 denaturing PAGE (Fig. 4a; left gel). We first examined the 3TAintra system. Lane 2 contains the crystal in the absence of UV and only the denatured single strands of the tile can be seen. By contrast, two retarded species are seen in lane 3; these correspond to the slower-mobility cross-linked products (ii) and (iii) 15 observed in the solution studies performed above. As before, the efficiency of cross-linking was improved by longer UV exposure or by increasing TFO concentration (Fig. S7). The same experiments were undertaken on the 3TS-intra tile and again cross-linking reactions did not differ from those seen in solution 20 experiments (Fig. 4a; middle gel).
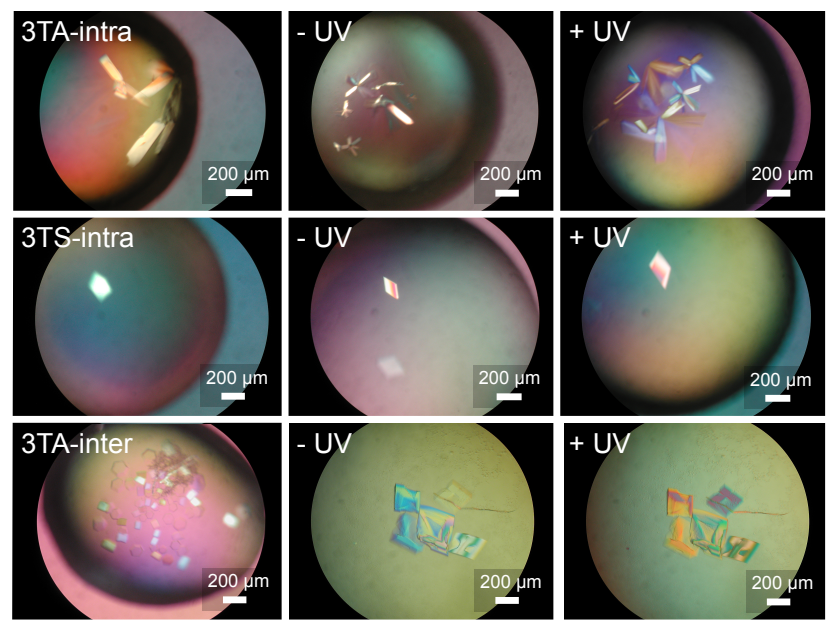

Fig. 3. Crystallisation and cross-linking of the tensegrity triangle crystal. Optical images of the tile in the absence and presence of TFO before and 25 after UV exposure. Tiles were annealed to $20{ }^{\circ} \mathrm{C}$ before addition of the TFO and the setup left to equilibrate at $4{ }^{\circ} \mathrm{C}$ until crystals were observed (3-5 days). The images were taken in the presence of polarizer.

We then examined the ability of the psoralen TFO to direct the cross-linking of a TpA step generated only upon the association 30 of consecutive tiles within the crystal using the 3TA-inter tile. This was enabled by positioning the oligopurine target sequence along a region of the non-crossover strand that spanned both the crossover junction $^{8}$ and the intermolecular contact involving the sticky-ends (Fig. 1; inset). Separating the products of cross35 linking by denaturing PAGE revealed two species with a slower mobility than those observed in the absence of cross-linking (Fig. 4a; right gel). These species are shorter than those obtained for the 3TA-intra and 3TS-intra systems on account of the crosslinking occurring between the helical and shorter crossover 40 strands of the tiles located at the corners. As above the intensity of the band for the species with slower mobility, attributable to the bis-adduct, is greater than the intensity of the band for the faster migrating species, attributable to the mono-adduct. This demonstrates that the psoralen-TFO can be used to direct efficient
${ }_{45}$ cross-linking between adjacent tiles within the crystal.

Lastly, we examined whether cross-linking improved the thermal stability of the DNA crystals. Drops containing crystals in the presence of TFO before and after UV exposure were heated at a rate of $0.2^{\circ} \mathrm{C} / \mathrm{min}$ using a temperature-programmed incubator 50 and crystal stability observed using a microscope (Fig. 4b; S8S10). We considered the crystals melted at the point at which that they were no longer visible to the naked eye. The crystal melting temperatures for the 3TA-intra, 3TS-intra and 3TA-inter crystals with TFO but in the absence of UV exposure were 26, 27 and 23

${ }_{55}{ }^{\circ} \mathrm{C}$, respectively. By contrast, the melting temperatures measured after UV irradiation were 27,28 and $31{ }^{\circ} \mathrm{C}$, increases of 1,1 and $8{ }^{\circ} \mathrm{C}$, respectively. As expected, cross-linking regions that do not span the intermolecular contacts between triangles of the 3TAintra and 3TS-intra systems had only a small influence on crystal 60 stability. By contrast, cross-linking regions that span the intermolecular contacts at each end of a single helix within the triangle of the 3TA-inter system resulted in a dramatic increase in crystal stability (Fig. 4b). Although the crystals generated by the 3TS-intra and 3TA-inter triangles exhibit similar melting ${ }_{65}$ temperatures after cross-linking, the 3TA system is inherently less stable than the 3TS system before cross-linking on account of the difference in nucleotide sequences between the two triangles, particularly at the sticky-ends. Consequently, it is likely that the stability of the 3TA-inter system could be improved further by 70 alterations to sequence design, i.e., increasing GC-content, optimising base stacking interactions, etc. Moreover, since the experiments before and after cross-linking were undertaken in the presence of the TFO, the increase in thermal stability was due to covalent cross-linking and not simply through the non-covalent 75 binding of the TFO across the sticky-ends. ${ }^{10 \mathrm{~b}}$ Crystal stability might also be improved by designing a system that cross-links the intermolecular contacts at each end of all three helices within the triangle.

a

a (i)

(ii)

(iii)

b

3TA-inter
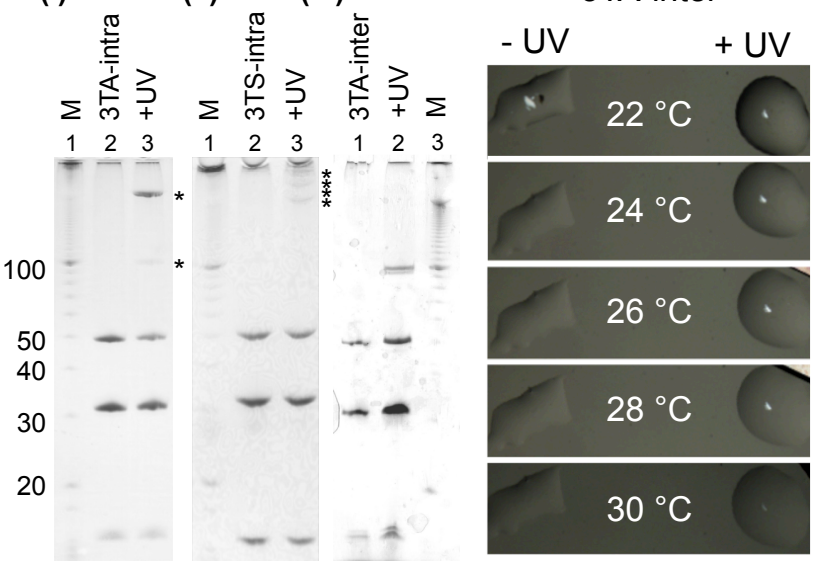

Fig. 4. Characterising cross-linking within tensegrity triangle crystals. (a) Denaturing PAGE analysis of cross-linked crystals. Cross-linked products highlighted with an asterisk are identical to those obtained from the same 85 tiles in solution. (b) Thermal stability of the cross-linked 3TA-inter crystal. Drops containing the crystal before (left) and after cross-linking (right) were heated using a temperature-programmed incubator. The images were taken in the absence of polarizer. 
In summary, we have shown that triplex formation can be used to direct photo-cross-linking reactions within or between the tiles of tensegrity triangle crystals. Cross-linking can be achieved either before or after crystal assembly. Here we have used TFOs 5 containing a single psoralen modification attached to the 5 '-end of the TFO, though TFOs containing single or multiple psoralen modifications are synthetically available. ${ }^{9}$ TFOs could therefore be used to direct two or more cross-linking reactions to a single binding site that is embedded at any position along the helices 10 within the crystal, i.e., between the crossovers of the triangle and/or across the complementary overhangs between tiles. ${ }^{10 a, b}$ Such covalently closed crystals may prove useful for applications that entail removal of the crystals from their mother liquor.

\section{Notes and references}

This work was supported from BBSRC grant BB/H019219/1 to DAR, KRF and TB and the following grants to NCS: GM-29554 from the National Institute of General Medical Sciences, grants EFRI-1332411, CCF-1117210 and and CCF-1526650 from the National Science 20 Foundation, MURI W911NF-11-1-0024 from the Army Research Office, grants N000141110729 and N000140911118 from the Office of Naval Research, DE-SC0007991 from the Department of Energy for DNA synthesis and partial salary support, and grant GBMF3849 from the Gordon and Betty Moore Foundation.

${ }_{25}{ }^{a}$ Department of Chemistry, New York University, New York, NY 10003, USA.Tel:+001212-998-8395; E-mail: ned.seeman@nyu.edu

${ }^{b}$ The RNA Institute, University at Albany, State University of New York, Albany, New York, 12222, USA.

${ }^{c}$ Centre for Biological Sciences, University of Southampton,

30 Southampton, Hampshire, SO17 1BJ, UK. Tel: +044 2380-598413; Email:d.a.rusling@soton.ac.uk.

${ }^{d}$ Department of Chemistry, University of Oxford, Chemistry Research Laboratory, 12 Mansfield Road, Oxford, OXI 3QZ, UK

${ }^{e}$ Department of Chemistry, Purdue University, West Lafayette, IN 47907, 35 USA.

$\dagger$ Electronic Supplementary Information (ESI) available: [Full experimental methods, Figures S1-10].

1. (a) D. Liu, M. Wang, Z. Deng, R. Walulu and C. Mao, J. Am. Chem. Soc., 2004, 126, 2324. (b) J. Zheng, J. J. Birktoft, Y. Chen, T. Wang,

40 R. Sha, P. E. Constantinou, S. L. Ginell, C. Mao and N. C. Seeman, Nature, 2009, 461, 74.

2. (a) B. H. Robinson and N. C. Seeman, Protein Engineering, 1987, 1 , 295. (b) O. Wilner, Y. Weizmann and R. Gill, Nature Nanotech., 2009, 4, 249. (c) N. C. Seeman, J. Theor. Biol. 1982, 99, 237.

45 3. E. Sage and E. Moustacchi, Biochemistry, 1987, 26, 3307.

4. A. Rajendran, M. Endo, Y. Katsuda, K. Hidaka and H. Sugiyama, $J$. Am. Chem. Soc. 2011, 133, 14488.

5. (a) H. E. Moser and P. B. Dervan, Science, 1987, 238, 645; (b) T. Le Doan, L. Perrouault, D. Praseuth, N. Habhoub, J. L. Decout, N. T.

50 Thuong, J. Lhomme and C. Hélène, Nucleic Acids Res., 1987, 19, 7749. (c) K. R. Fox, Curr. Med. Chem., 2000, 7, 17.

6. D. A. Rusling, A. R. Chandrasekaran, Y. P. Ohayon, T. Brown, K. R. Fox, R. Sha, C. Mao and N. C. Seeman, Angew. Chem. Int. Ed., 2014, 53, 3979.

55 7. M. Takasugi, A. Guendouz, M. Chassignol, J. L. Decout, J. Lhomme, N. T. Thuong and C. Hélène, Proc. Natl. Acad. Sci. U. S. A., 1991, 88,5602 .

8. D. A. Rusling, I. S. Nandhakumar, T. Brown and K. R. Fox. ACS Nano, 2012, 6, 3604.

60 9. H. Li, V. J. Broughton-Head, G. Peng, V. E. Powers, M. J. Ovens, K. R. Fox and T. Brown, Bioconjug Chem., 2006, 17, 1561.

10. (a) D. A. Rusling, I. Nandhakumar, T. Brown and K. R. Fox. Chem Comm., 2012, 48, 9592. (b) J. Zhao, A. R. Chandrasekaran, Q. Li, X. Li, R. Sha, N. C. Seeman and C. Mao. Angew. Chem. Int. Ed., 2015, $54,9936$.

4 I Journal Name, [year], [vol], @๑-๑๑This journal is @ The Royal Society of Chemistry [year] 